\title{
On Ramsey $\left(\mathrm{mK}_{\mathbf{2}}, P_{\mathbf{4}}\right)$-Minimal Graphs
}

\author{
Asep Iqbal Taufik ${ }^{1}$, Denny Riama Silaban ${ }^{1, *}$, Kristiana Wijaya ${ }^{2}$
}

\author{
${ }^{I}$ Department of Mathematics, Faculty of Mathematics and Natural Sciences \\ Universitas Indonesia, Depok 16424, Indonesia \\ ${ }^{2}$ Graph, Combinatorics, and Algebra Research Group, Department of Mathematics, \\ Faculty of Mathematics and Natural Sciences, Universitas Jember, Jember 68121, Indonesia \\ *Corresponding author. Email: denny@sci.ui.ac.id
}

\begin{abstract}
Let $F, G$, and $H$ be simple graphs. The notation $F \rightarrow(G, H)$ means that any red-blue coloring of all edges of $F$ will contain either a red copy of $G$ or a blue copy of $H$. Graph $F$ is a Ramsey $(G, H)$-minimal if $F \rightarrow(G, H)$ but for each $e$ $\in E(F),(F-e) \nrightarrow(G, H)$. The set $\mathcal{R}(G, H)$ consists of all Ramsey $(G, H)$-minimal graphs. Let $m K_{2}$ be matching with m edges and $P_{n}$ be a path on $\mathrm{n}$ vertices. In this paper, we construct all disconnected Ramsey minimal graphs, and found some new connected graphs in $\mathcal{R}\left(3 K_{2}, P_{4}\right)$. Furthermore, we also construct new Ramsey minimal graphs in $\mathcal{R}((m+$ 1) $\left.K_{2}, P_{4}\right)$ from Ramsey minimal graphs in $\mathcal{R}\left(m K_{2}, P_{4}\right)$ for $m \geq 4$, by subdivision operation.
\end{abstract}

Keywords: Matching, Path, Ramsey minimal graphs, Subdivision.

\section{INTRODUCTION}

Let $F, G$, and $H$ be simple graphs. The notation $F \rightarrow$ $(G, H)$ means that in any red-blue coloring of $F$, there exists a red copy of $G$ or a blue copy of $H$ as a subgraph. A $(G, H)$-coloring of $F$ is a red-blue coloring of $F$ such that neither a red $G$ nor a blue $H$ occurs. A graph $F$ is said to be a Ramsey $(G, H)$-minimal if $F \rightarrow(G, H)$ but for any $e \in E(F)$, there exists a $(G, H)$-coloring on graph $F-e$. The set of all Ramsey $(G, H)$-minimal graphs is denoted by $\mathcal{R}(G, H)$.

The determination and the characterization of all graphs $F$ belonging to $\mathcal{R}(G, H)$ are the main problems in Ramsey $(G, H)$-minimal graphs. Some papers discuss the problem of determining all graphs in $\mathcal{R}(G, H)$. Burr et al. [1] proved that if $H$ is any graph then $\mathcal{R}\left(m K_{2}, H\right)$ is a finite set. One of challenging problems in Ramsey Theory is to characterize all graphs in the set $\mathcal{R}\left(m K_{2}, H\right)$ for a given graph $H$.

Let $K_{n}, C_{n}$, and $P_{n}$ be a complete graph, a cycle, and a path on $n$ vertices, respectively. The characterization of Ramsey minimal graphs belonging to $\mathcal{R}\left(2 K_{2}, K_{4}\right)$ can be seen in [2, 3]. The set $\mathcal{R}\left(2 K_{2}, P_{3}\right)$ is determined by Mengersen and Oeckermann [4]. Mushi and Baskoro [5] determined all graphs in $\mathcal{R}\left(3 K_{2}, P_{3}\right)$. Furthermore, the set $\mathcal{R}\left(4 K_{2}, P_{3}\right)$ given by Wijaya et al. [6].

Wijaya et al. [7] showed that the cycle $C_{s}$ belongs to $\mathcal{R}\left(m K_{2}, P_{n}\right)$ if and only if $s \in[m n-n+1 \leq s \leq$ $m n-1$ ]. Recently Wijaya et al. [8] constructed a family of Ramsey $\left(m K_{2}, P_{4}\right)$ minimal graphs from Ramsey $\left((m-1) K_{2}, P_{4}\right)$ minimal graph by doing 4 times subdivision on any edge belongs to a cycle in a Ramsey $\left(m K_{2}, P_{4}\right)$-minimal graph. Furthermore, Wijaya et al. [9] constructed a class of disconnected Ramsey $\left(m K_{2}, H\right)$ minimal graphs from a union of two or more connected graphs. Motivated by result in [9], in this paper, we focus on determining all disconnected graphs in $\mathcal{R}\left(3 K_{2}, P_{4}\right)$, and found some connected graphs belonging to Ramsey $\left(3 K_{2}, P_{4}\right)$-minimal. In addition, we also construct some graph in $\mathcal{R}\left((m+1) K_{2}, P_{4}\right)$ by doing subdivisions to graphs in $\mathcal{R}\left(m K_{2}, P_{4}\right)$ for $\mathrm{m} \geq 4$.

\section{PRELIMINARIES}

Let $G=(V, E)$ be graph. If $U \subseteq V$, then $G-U$ is a graph obtained from $G$ by deleting vertices in $U$ and all incident edges. If $H \subseteq G$, then $G-E(H)$ is a graph obtained from $G$ by deleting edges in $H$. When $U=\{v\}$ and $E(H)=\{e\}$, for simplicity, we write $G-v$ and $G-$ $e$, respectively.

Lemma 1 and 2 provide the necessary and sufficient conditions for any graph in $\mathcal{R}\left(3 K_{2}, H\right)$ for any graph $H$.

Lemma 1. [9, 10] For any fixed graph $H$, the graph $F \rightarrow$ $\left(3 K_{2}, H\right)$ holds if and only if the following four conditions are satisfied: (i) $F-\{u, v\} \supseteq H$ for each $u, v \in V(F)$, (ii) $F-u-E\left(K_{3}\right) \supseteq H$ for each $u \in V(F)$ 
and a triangle $K_{3}$ in $F$, (iii) $F-E\left(2 K_{3}\right) \supseteq H$ for every two triangles in $F$, (iv) $F-E\left(S_{5}\right) \supseteq H$ for every induced subgraph with 5 vertices $S$ in $F$.

Lemma 2. [9, 10] Let $H$ be a simple graph. Suppose $F$ is a Ramsey $\left(3 K_{2}, H\right)$-graph. $F$ is said to be minimal if for each $e \in E(F)$ satisfy $(F-e) \nrightarrow\left(3 K_{2}, H\right)$, that is (i) $(F-e)-\{u, v\} \nsupseteq H$ for each $u, v \in V(F)$, ii) $F-$ $u-E\left(K_{3}\right) \nsupseteq H$ for each $u \in V(F)$ and a triangle $K_{3}$ in $F$, (iii) $F-E\left(2 K_{3}\right) \nsupseteq H$ for every two triangles in $F$, (iv) $F-E\left(S_{5}\right) \nsupseteq H$ for every induced subgraph with 5 vertices $S$ in $F$.

Any graph satisfying all conditions in Lemma 1 and 2 is a Ramsey $\left(3 K_{2}, H\right)$-minimal graph. The condition stated in Lemma 2 is called the minimality property of a graph in $\mathcal{R}\left(3 K_{2}, H\right)$. In [10], Wijaya et al. defined $S F(e, t)$ as a $t$ times subdivision of edge $e$ in the connected graph $F$, and gave Theorem 3. Moreover, Baskoro and Yulianti [7] gave Theorem 4.

Theorem 3. Let $F$ be a connected graph and $m \geq 2$ be an integer. Suppose $\alpha$ is one non-pendant edge of $F$. If $F \in$ $\mathcal{R}\left(m K_{2}, P_{4}\right)$, then $S F(\alpha, 4) \in \mathcal{R}\left((m+1) K_{2}, P_{4}\right)$.

Theorem 4. [7] $\mathcal{R}\left(2 K_{2}, P_{4}\right)=\left\{2 P_{4}, C_{7}, C_{6}, C_{5}, C_{4}^{+}\right\}$, where $C_{4}^{+}$is a $C_{4}$ with additional two pendant vertices as in Figure 1

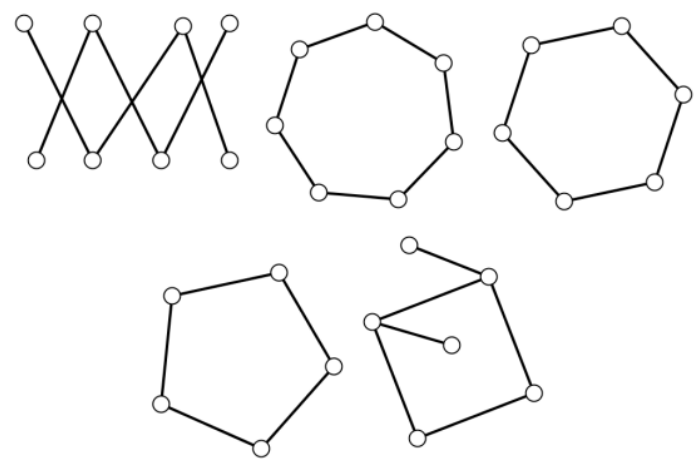

Figure 1 All graphs in $\mathcal{R}\left(2 K_{2}, P_{4}\right)$.

\section{MAIN RESULTS}

\subsection{Disconnected Graph in $\mathcal{R}\left(3 K_{2}, P_{4}\right)$}

In this section, we give all disconnected graphs belonging to $\mathcal{R}\left(3 K_{2}, P_{4}\right)$.

Theorem 5. $G \cup P_{4} \in \mathcal{R}\left(3 K_{2}, P_{4}\right)$ if and only if $G \in$ $\mathcal{R}\left(2 K_{2}, P_{4}\right)$.

Proof. $(\Leftarrow)$ We will show that for any $G \in \mathcal{R}\left(2 K_{2}, P_{4}\right)$, then $G \cup P_{4} \in \mathcal{R}\left(3 K_{2}, P_{4}\right)$. Since $G \in \mathcal{R}\left(2 K_{2}, P_{4}\right)$, then $G \rightarrow\left(2 K_{2}, P_{4}\right)$ and $G-e \rightarrow\left(2 K_{2}, P_{4}\right)$ for any $e \in E(G)$. Since $G \rightarrow\left(2 K_{2}, P_{4}\right)$, by coloring all edges incident to any vertex in $G$ produces a blue copy of $P_{4}$ subset of $G$. Thus, any red coloring of two independent edges in $G$ produces blue copy of $P_{4}$ subset of $G \cup P_{4}$. Moreover, any red coloring of one edge in $G$ and one edge in $P_{4}$ produces a blue copy of $P_{4}$ subset of $G \cup P_{4}$. Hence, $G \cup$ $P_{4} \rightarrow\left(3 K_{2}, P_{4}\right)$. Let $e_{1} \in E(G)$ and $e_{2} \in E\left(P_{4}\right)$. Since $G-e_{1} \nrightarrow\left(2 K_{2}, P_{4}\right)$, there exists a red-blue coloring of $G-e_{1}$ where a red $K_{2}$ occurs and blue $P_{4}$ cannot be found. Therefore, there exists a red-blue coloring on $G \mathrm{U}$ $P_{4}-e_{1}$ where neither a red $3 K_{2}$ nor a blue $P_{4}$ occurs. Moreover, any red coloring of two independent edges in $G \subset G \cup P_{4}-e_{2}$ produces red-blue coloring of $G \cup P_{4}-$ $e_{2}$ where neither a red $3 K_{2}$ nor a blue $P_{4}$ occurs. Hence, $G \cup P_{4}-e \rightarrow\left(3 K_{2}, P_{4}\right)$. Since $G \cup P_{4} \rightarrow\left(3 K_{2}, P_{4}\right)$ and $G \cup P_{4}-e \nrightarrow\left(3 K_{2}, P_{4}\right)$ for any $e \in E(G)$, then $G \cup$ $P_{4} \in \mathcal{R}\left(3 K_{2}, P_{4}\right)$.

$(\Rightarrow)$ If $G \cup P_{4} \in \mathcal{R}\left(3 K_{2}, P_{4}\right)$, then $G \in \mathcal{R}\left(2 K_{2}, P_{4}\right)$. For a contradiction, suppose that $G \notin \mathcal{R}\left(2 K_{2}, P_{4}\right)$. Then, we have two cases.

Case 1. Suppose $G \rightarrow\left(2 K_{2}, P_{4}\right)$. Then there exist a $\left(2 K_{2}, P_{4}\right)$-coloring of $G$. Extend the coloring to color $G \cup P_{4}$ and color the edges of $P_{4}$ by red. Thus, there exist a $\left(3 K_{2}, P_{4}\right)$-coloring of $G \cup P_{4}$, which contradicts the fact that $G \cup P_{4} \in \mathcal{R}\left(3 K_{2}, P_{4}\right)$.

Case 2. Suppose $G \rightarrow\left(2 K_{2}, P_{4}\right)$, but $G$ is not minimal. It means there exists a graph $H \in \mathcal{R}\left(2 K_{2}, P_{4}\right)$ where $G \supset$ $H$. Thus $G \cup P_{4} \supset H \cup P_{4}$. Since $H \in \mathcal{R}\left(2 K_{2}, P_{4}\right)$, then $H \cup P_{4} \in \mathcal{R}\left(3 K_{2}, P_{4}\right)$ by the first case, which contradicts to the minimality of $G \cup P_{4}$.

Therefore, from two cases above, we conclude that $G \cup P_{4} \in \mathcal{R}\left(3 K_{2}, P_{4}\right)$ if and only if $G \in \mathcal{R}\left(2 K_{2}, P_{4}\right)$.

Theorem 6. Let $H$ be a disconnected graph in $\mathcal{R}\left(3 K_{2}, P_{4}\right)$. Therefore, one component of $H$ must be isomorphic to $P_{4}$.

Proof. Suppose to the contrary that $H=H_{1} \cup H_{2}$ and none of $H_{1}$ or $H_{2}$ is isomorphic to $P_{4}$. Since there is no component in $H$ isomorphic to $P_{4}$, there is no component $P_{4}$ in either $H_{1}$ and $H_{2}$. Every vertex in $H$ is in a connected subgraph containing a $P_{4}$. Then, both $H_{1}$ and $H_{2}$ contain $P_{4}$. Therefore, there will be edges $e_{1} \in E\left(H_{1}\right)$ and $e_{2} \in E\left(H_{2}\right)$ such that $P_{4} \subseteq H_{1}-e_{1}$ and $P_{4} \subseteq$ $H_{2}-e_{2}$. Since $H \in \mathcal{R}\left(3 K_{2}, P_{4}\right)$, there exist a $\left(3 K_{2}, P_{4}\right)$ coloring of $H-e_{1}$ and $H-e_{2}$, say $J_{1}$ and $J_{2}$, respectively. Under $J_{1}, H_{1}-e_{1}$ must contain at least one red edge and $\mathrm{H}_{2}$ must have a $\left(2 K_{2}, P_{4}\right)$-coloring. Since if it is not the case, $H-e_{1}$ would contain a red $3 K_{2}$ or blue $P_{4}$, a contradiction to the minimality of $H$. Moreover, under $J_{2}, H_{2}-e_{2}$ must contain at least one red edge and $H_{1}$ must have a $\left(2 K_{2}, P_{4}\right)$-coloring. We conclude that we will obtain a $\left(3 K_{2}, P_{4}\right)$-coloring of $H$ if we color $H$ by using $J_{1}$ on $H_{2}$ and $J_{2}$ on $H_{1}$, which contradicts to the minimality of $H$.

Therefore, if $H$ is a disconnected graph in $\mathcal{R}\left(3 K_{2}, P_{4}\right)$. Then, one component of $H$ must be isomorphic to $P_{4}$. 
Theorem 7. The graphs $C_{5} \cup P_{4}, C_{6} \cup P_{4}, C_{7} \cup P_{4}$, $C_{4}^{+} \cup P_{4}$ and $3 P_{4}$ are the only disconnected graphs in $\mathcal{R}\left(3 K_{2}, P_{4}\right)$.

Proof. Using Theorem 6, if $F$ is a disconnected graph in $\mathcal{R}\left(3 K_{2}, P_{4}\right)$, then $F$ must have a component isomorphic to $P_{4}$. Furthermore, Theorem 5 states that the other component of $F$ must be a member of the set $\mathcal{R}\left(2 K_{2}, P_{4}\right)$. Moreover, Theorem 4 determined all graphs in $\mathcal{R}\left(2 K_{2}, P_{4}\right)$. Therefore, the graphs $C_{5} \cup P_{4}, C_{6} \cup P_{4}, C_{7} \cup$ $P_{4}, C_{4}^{+} \cup P_{4}$ and $3 P_{4}$ are the only disconnected graphs in $\mathcal{R}\left(3 K_{2}, P_{4}\right)$.

\subsection{Some Connected Graphs in $\mathcal{R}\left(3 \mathrm{~K}_{2}, P_{4}\right)$}

In this section, we determine some connected graphs other than the cycle belonging to $\mathcal{R}\left(3 K_{2}, P_{4}\right)$. First, we show that a graph $F_{1}$, depicted in Fig. 2, is a Ramsey $\left(3 K_{2}, P_{4}\right)$-minimal graph.

Proposition 8. Let $F_{1}$ be a graph as depicted in Fig. 2 . The graph $F_{1}$ is a Ramsey $\left(3 K_{2}, P_{4}\right)$-minimal graph.

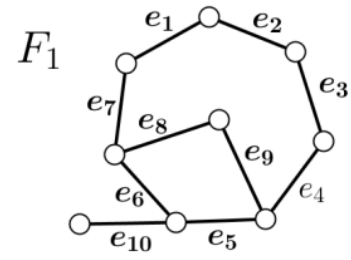

Figure 2 The graph $F_{1} \in\left(3 K_{2}, P_{4}\right)$.

Proof. First, we show that for any red-blue coloring of $F_{1}$ contains a red $3 K_{2}$ or a blue $P_{4}$. We can see that $F_{1}-$ $\{u, v\}$ always contains a path $P_{4}$ for any $u, v \in V\left(F_{1}\right)$. It can be verified that $F_{1}-E\left(S_{5}\right) \supseteq H$ for every induced subgraph with 5 vertices $S$ in $F_{1}$. Since $F_{1}$ has no triangle, then by Lemma 1 we have that $F_{1} \rightarrow\left(3 K_{2}, P_{4}\right)$. Next, we prove the minimality property. For any edge $e$ we will show that $\left(F_{1}-e\right) \nrightarrow\left(3 K_{2}, P_{4}\right)$. If $e$ is one of the dashed edges in Fig. 3, then each redblue coloring in Fig. 3 is the $\left(3 K_{2}, P_{4}\right)$-coloring on $F_{1}-$ $e$. Therefore $F_{1} \in \mathcal{R}\left(3 K_{2}, P_{4}\right)$.
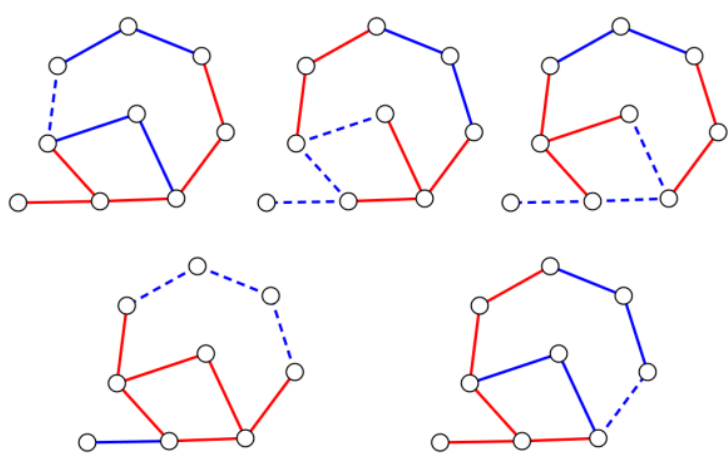

Figure 3 The $\left(3 K_{2}, P_{4}\right)$-colorings on $F_{1}-e$ if $e$ is one of the dashed edges.
Suppose $V\left(C_{n}\right)=\left\{v_{1}, v_{2}, \ldots, v_{n-1}, v_{n}\right\}$ is the vertex-set of $C_{n}$. We define a graph $C_{n}^{a}$ as a graph obtained from $C_{n}$ by adding a pendant vertex, say $v_{n+1}$, adjacent to $v_{a}$ for $a \in[1, n]$. A graph $C_{n}^{a, b}$ is obtained from $C_{n}$ by adding two pendant vertices, say $v_{n+1}$ and $v_{n+2}$, adjacent to $v_{a}$ and $v_{b}$, respectively, for $a, b \in$ $[1, n]$. Moreover, following Wijaya et al. in [10], we define special graphs with certain circumference. Let $a, b, c, d, e, f$, $g$ and $h$ be eight integers. Graph $C_{n}[(a, b)$, $(c, d)$ ] is obtained from $C_{n}$ by adding two new edges $v_{a} v_{b}$ and $v_{c} v_{d}$. Graph $C_{n}[(a, b),(c, d),(e, f)]$ is obtained from $C_{n}$ by adding three new edges $v_{a} v_{b}$, $v_{c} v_{d}$, and $v_{e} v_{f}$. Graph $C_{n}[(a, b),(c, d),(e, f),(g, h)]$ is obtained from $C_{n}$ by adding four new edges $v_{a} v_{b}$, $v_{c} v_{d}, v_{e} v_{f}$, and $v_{g} v_{h}$. Now, consider graphs $C_{6}[(1,4),(2,5),(2,6),(3,5)], \quad C_{7}^{5}[(1,3),(2,6),(5,7)]$ $C_{7}[(1,5),(3,7)], C_{7}^{7}[(2,6),(3,7)], C_{8}[(2,7),(4,7),(6,8)]$ $C_{6}^{3,4}[(1,4),(3,6)]$ as depicted in Fig. 4. We will show that those graphs are Ramsey $\left(3 K_{2}, P_{4}\right)$-minimal.

Theorem 9. All graphs in Fig. 4 are Ramsey $\left(3 K_{2}, P_{4}\right)$ minimal graphs.

Proof. Let $F$ be any graph in Fig. 4. It is easy to see that $F$ satisfies all the conditions in Lemma 1 . Then, $F \rightarrow$ $\left(3 K_{2}, P_{4}\right)$ holds. Now, we will show the minimality property of $F$. Let $e$ be any edge in $F$. If $e$ is one of the dashed edges, then a $\left(3 K_{2}, P_{4}\right)$-coloring on $F-e$ is provided in Figures 5, 6, 7. 8, 9 and 10 respectively for all cases.
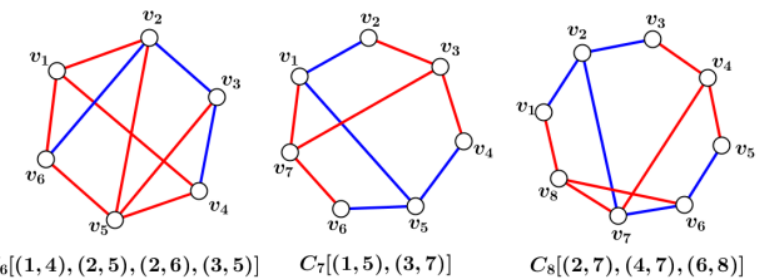

$C_{6}[(1,4),(2,5),(2,6),(3,5)]$

$C_{8}[(2,7),(4,7),(6,8)]$

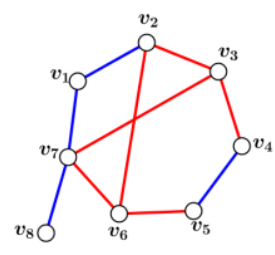

$C_{7}^{7}[(2,6),(3,7)]$

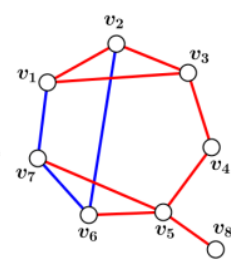

$C_{7}^{5}[(1,3),(2,6),(5,7)]$

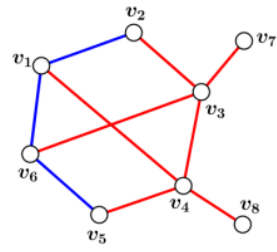

$C_{6}^{3,4}[(1,4),(3,6)]$
Figure 4 Six non-isomorphic graphs belonging to $\mathcal{R}\left(3 K_{2}, P_{4}\right)$ which is obtained from $C_{n}$ with some cords or pendant vertices or combination both. 

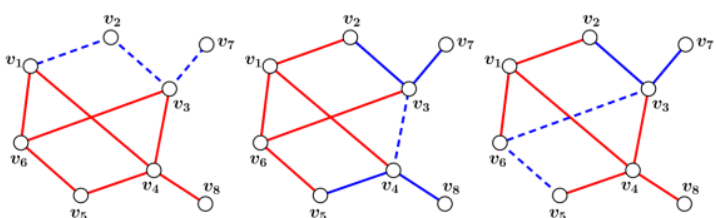

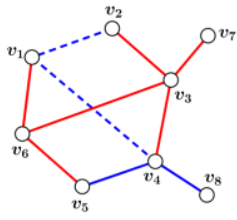
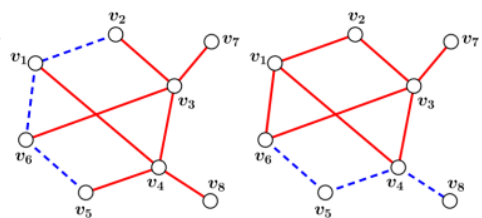

Figure 5 The $\left(3 K_{2}, P_{4}\right)$-colorings on $C_{6}^{3,4}[(1,4),(3,6)]-$ $e$ if $e$ is one of the dashed edges.
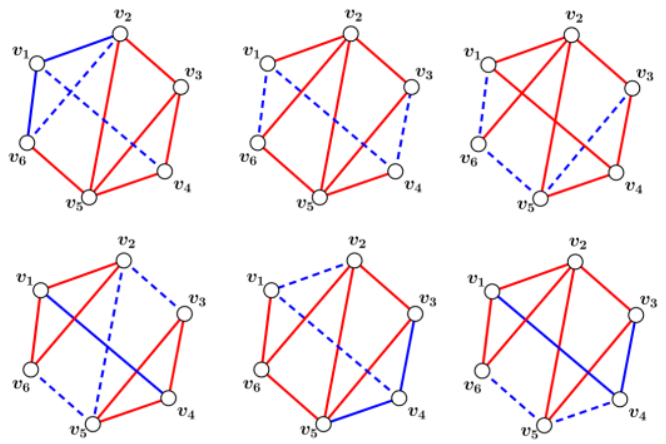

Figure 6 The $\left(3 K_{2}, P_{4}\right)$-colorings on $C_{6}[(1,4),(2,5),(2,6),(3,5)]-e$ if $e$ is one of the dashed edges.
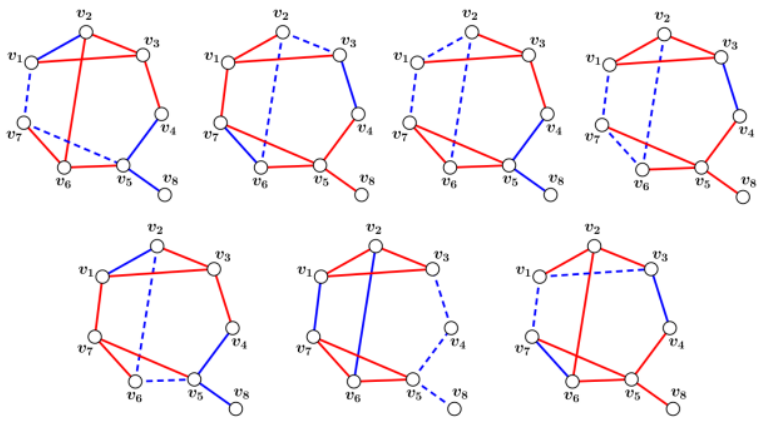

Figure 7 The $\left(3 K_{2}, P_{4}\right)$-colorings on $C_{7}^{5}[(1,3),(2,6),(5,7)]-e$ if $e$ is one of the dashed edges.

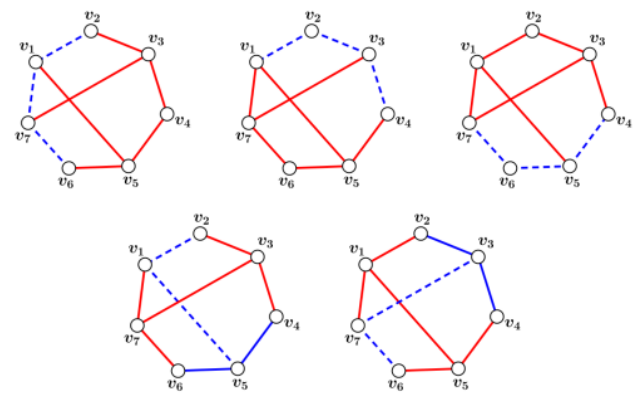

Figure 8 The $\left(3 K_{2}, P_{4}\right)$-colorings on $C_{7}[(1,5),(3,7)]-e$ if $e$ is one of the dashed edges.
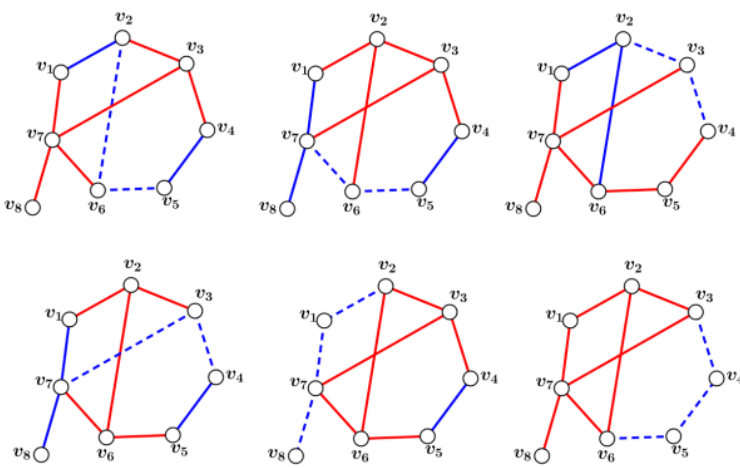

Figure 9 The $\left(3 K_{2}, P_{4}\right)$-colorings on $C_{7}^{7}[(2,6),(3,7)]-e$ if $e$ is one of the dashed edges.
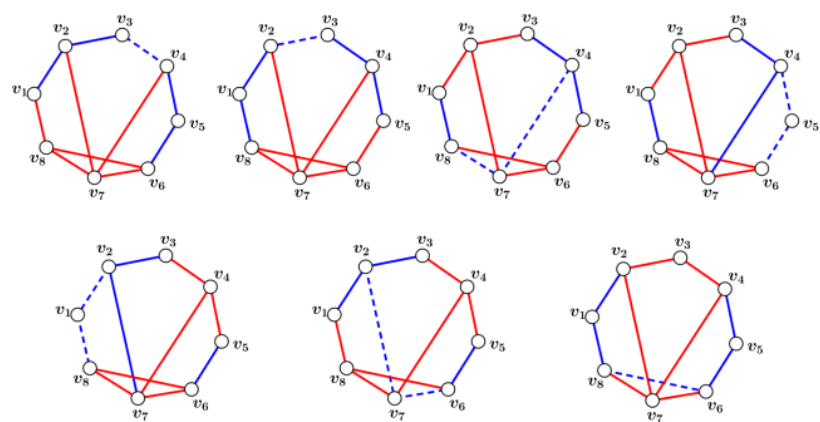

Figure $\mathbf{1 0}$ The $\left(3 K_{2}, P_{4}\right)$-colorings on $C_{8}[(2,7),(4,7),(6,8)]-e$ if $e$ is one of the dashed edges.

\subsection{Some New Family of Ramsey $\left(\mathrm{mK}_{2}, \mathrm{P}_{4}\right)$ - Minimal Graphs}

Recall that $S F(e, t)$ is a subdivision $t$ times of edge $e$. In the previous section, it has been shown that $F_{1} \in$ $\mathcal{R}\left(3 K_{2}, P_{4}\right)$. According to Theorem 3 , if we subdivide (4 times) any non-pendant edge of $F_{1}$, then we obtain three non-isomorphism graphs belonging to $\mathcal{R}\left(4 K_{2}, P_{4}\right)$, namely $S F_{1}\left(e_{1}, 4\right), \quad S F_{1}\left(e_{5}, 4\right)$, and $S F_{1}\left(e_{8}, 4\right)$ as depicted in Fig.11 (4 vertices, green vertex). The proof of the minimality of a graph $S F_{1}\left(e_{5}, 4\right)$ can be seen in Fig.12, while the minimality of the other graphs can be represented in the same way.
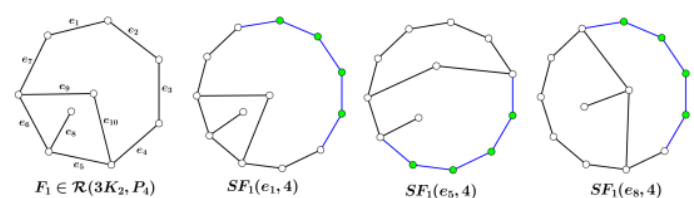

Figure 11 Three non-isomorphism graphs belonging to $\left(3 K_{2}, P_{4}\right)$ are obtained by subdividing four times ( 4 green vertices) a non-pendant edge of $F_{1}$. 


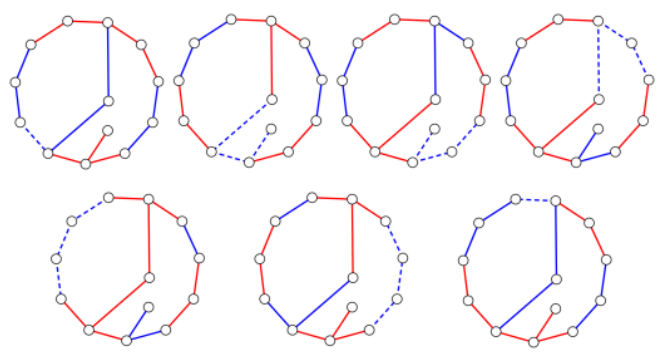

Figure 12 The $\left(4 K_{2}, P_{4}\right)$-colorings on $S F_{1}\left(e_{5}, 4\right)-e$ if $e$ is one of the dashed edges.

Now, we consider graph $C_{7}[(1,5),(3,7)]$. Since every edge in $C_{7}[(1,5),(3,7)]$ is non-pendant, then according to Theorem 3 , the subdivision (4 times) on any edge of $C_{7}[(1,5),(3,7)]$ will produce three non-isomorphism graphs in $\mathcal{R}\left(4 K_{2}, P_{4}\right)$. By repeating this process for the resulting graphs, we obtain Corollary 10.

Corollary 10. Let $m \geq 4$ be an integer. Then, the graphs $C_{4 m-5}[(1,5),(3,7)], C_{4 m-5}[(1,4 m-7),(4 m-9,4 m-$ $5)]$, and $C_{4 m-5}[(1,4 m-7),(3,4 m-5)]$ are in $\mathcal{R}\left(m K_{2}, P_{4}\right)$.

Proof. Let $\left\{v_{1}, v_{2}, \ldots, v_{7}\right\}$ be the vertex-set of $C_{7}[(1,5),(3,7)]$. The subdivision (4 vertices) on the edge $e=v_{1} v_{2}$ will result $C_{11}[(1,9),(7,11)]$. Since $C_{7}\left[(1,5),(3,7) \in \mathcal{R}\left(3 K_{2}, P_{4}\right)\right.$, then by Theorem 3 , we have that $C_{11}[(1,9),(7,11)] \in \mathcal{R}\left(4 K_{2}, P_{4}\right)$. Furthermore, by subdividing ( 4 vertices) the edge $e=v_{1} v_{2}$ of $C_{11}[(1,9),(7,11)]$, we obtain $C_{15}[(1,13),(11,15)]$. By Theorem 3, we have that $C_{15}[(1,13),(11,15)] \in$ $\mathcal{R}\left(5 K_{2}, P_{4}\right)$. By continuing this process and applying it to the resulting graph, then we obtain the graph $C_{4 m-5}[(1,4 m-7),(4 m-9,4 m-5)]$. By Theorem 3, $C_{4 m-5}[(1,4 m-7),(4 m-9,4 m-5)] \in \mathcal{R}\left(m K_{2}, P_{4}\right)$.

Next, by subdivision (4 vertices) on the edge $e=v_{3} v_{4}$ of the graph $C_{7}[(1,5),(3,7)]$, repeatedly, and apply Theorem 3, we obtain $C_{4 m-5}[(1,4 m-7),(3,4 m-$ $5)] \in \mathcal{R}\left(m K_{2}, P_{4}\right)$. By doing the same way to the edge $e=v_{7} v_{1}$, we obtain $C_{4 m-5}[(1,5),(3,7)] \in \mathcal{R}\left(m K_{2}, P_{4}\right)$.

In the same way, we can construct some other graphs in $\mathcal{R}\left(\mathrm{mK}_{2}, P_{4}\right)$ from some graph in $\mathcal{R}\left(3 K_{2}, P_{4}\right)$, namely, $C_{6}^{3,4}[(1,4),(3,6)] \quad, \quad C_{6}[(1,4),(2,5),(2,6),(3,5)]$, $C_{7}^{5}[(1,3),(2,6),(5,7)], \quad C_{7}^{7}[(2,6),(3,7)], \quad$ and $C_{8}[(2,7),(4,7),(6,8)]$. Therefore, we have Corollary 11.

Corollary 11. Let $m \geq 4$ be an integer. Then the following 19 graphs are in $\mathcal{R}\left(m K_{2}, P_{4}\right)$.
1. $C_{4 m-6}^{3,4}[(1,4),(3,6)]$,
2. $C_{4 m-6}^{4 m-9,4 m-8}[(1,4 m-8),(4 m-9,4 m-6)]$,
3. $C_{4 m-6}^{3,4 m-8}[(1,4 m-8),(3,4 m-6)]$,
4. $C_{4 m-6}[(1,4 m-8),(4 m-10,4 m-7)$,
$(4 m-10,4 m-6),(4 m-9,4 m-7)]$,
5. $C_{4 m-6}[(1,4 m-8),(2,4 m-7),(2,4 m-6)$,

$(4 m-9,4 m-7)]$,

6. $C_{4 m-6}[(1,4),(2,5),(2,6),(3,5)]$,

7. $C_{4 m-5}^{7}[(2,6),(3,7)]$,

8. $C_{4 m-5}^{4 m-5}[(2,4 m-6),(4 m-9,4 m-5)]$,

9. $C_{4 m-5}^{4 m-5}[(2,4 m-6),(3,4 m-5)]$,

10. $C_{4 m-5}^{4 m-5}[(2,6),(3,4 m-5)]$

11. $C_{4 m-5}^{5}[(1,3),(2,6),(5,7)]$,

12. $C_{4 m-5}^{4 m-7}[(1,4 m-9),(4 m-10,4 m-6),(4 m-$ $7,4 m-5)]$,

13. $C_{4 m-5}^{4 m-7}[(1,4 m-9),(2,4 m-6),(4 m-7,4 m-$ 5)],

14. $C_{4 m-5}^{4 m-7}[(1,3),(2,4 m-6),(4 m-7,4 m-5)]$,

15. $C_{4 m-5}^{5}[(1,3),(2,4 m-6),(5,4 m-5)]$,

16. $C_{4 m-5}^{5}[(1,3),(2,6),(5,4 m-5)]$,

17. $C_{4(m-1)}[(2,7),(4,7),(6,8)]$,

18. $C_{4(m-1)}[(2,4 m-5),(4 m-8,4 m-5)$,

$(4 m-6,4(m-1))]$,

19. $C_{4(m-1)}[(2,7),(4,7),(6,4(m-1))]$.

\section{CONCLUSION}

In this paper, we discuss the construction of a disconnected Ramsey minimal graph in $\mathcal{R}\left(3 K_{2}, P_{4}\right)$ from Ramsey minimal graph ini $\mathcal{R}\left(2 K_{2}, P_{4}\right)$. We show that all disconnected graphs in $\mathcal{R}\left(3 K_{2}, P_{4}\right)$ are $C_{5} \cup P_{4}, C_{6} \cup P_{4}$, $C_{7} \cup P_{4}, C_{4}^{+} \cup P_{4}$, and $3 P_{4}$. In addition, we give some connected graphs in $\mathcal{R}\left(3 K_{2}, P_{4}\right)$, namely, $F_{1}$, $C_{6}[(1,4),(2,5),(2,6),(3,5)], \quad C_{7}^{5}[(1,3),(2,6),(5,7)]$ $C_{7}[(1,5),(3,7)], C_{7}^{7}[(2,6),(3,7)], C_{8}[(2,7),(4,7),(6,8)]$ $C_{6}^{3,4}[(1,4),(3,6)]$ as depicted in Fig. 4. Furthermore, we also construct nineteen new families of Ramsey $\left(m K_{2}, P_{4}\right)$ minimal graphs for $m \geq 4$.

\section{ACKNOWLEDGMENTS}

Part of this research is funded by PUTI KIUniversitas Indonesia 2020 Research Grant No. NKB779/UN2.RST/HKP.05.00/ 2020.

\section{AUTHORS' CONTRIBUTIONS}

Asep Iqbal Taufik: Conceived and designed experiments; Conducted experiments; Wrote the paper original draft preparation.

Denny Riama Silaban: Supervision and validation; Wrote the paper - review and editing.

Kristiana Wijaya: Supervision and validation; Wrote the paper - review and editing. 


\section{REFERENCES}

[1] S.A. Burr, P. Erdös, R.J. Faudree, R.H. Schelp, A class of Ramsey-finite graphs, Proc. 9th Conf. Combinatorics, Graph Theory, and Computing, pp. 171-180.

[2] E.T. Baskoro, K. Wijaya, On Ramsey $\left(2 K_{2}, K_{4}\right)$ minimal graphs, in: Mathematics in the $21 \mathrm{st}$ Century, Springer Proc. Math. Stat. 98 (2015) 1117.

[3] K. Wijaya, E.T. Baskoro, H. Assiyatun, D. Suprijanto, The complete list of Ramsey $\left(2 K_{2}, K_{4}\right)$ minimal graphs, Electron. J. Graph Theory Appl. 3 (2) (2015) 216-227.

DOI: https://doi.org/10.5614/ejgta.2015.3.2.9

[4] I. Mengersen, J. Oeckermann, Matching-star Ramsey sets, Discrete Appl. Math. 95 (1999) 417-424.

[5] H. Muhshi, E.T. Baskoro, On Ramsey $\left(3 K_{2}, P_{3}\right)-$ minimal graphs, AIP Conf. Proc. 1450 (2012) 110-117. DOI: https://doi.org/10.1063/1.4724125
[6] K. Wijaya, E.T. Baskoro, H. Assiyatun, D. Suprijanto, On Ramsey $\left(4 K_{2}, P_{3}\right)$-minimal graphs, AKCE Int. J. Graphs Comb. 15 (2018) 174-186. DOI: https://doi.org/10.1016/j.akcej.2017.08.003

[7] K. Wijaya, E.T. Baskoro, H. Assiyatun, D. Suprijanto, On unicyclic Ramsey $\left(m K_{2}, P_{3}\right)$ minimal graphs, Proc. Comput. Sci. 74 (2015) $10-14$.

DOI: https://doi.org/10.1016/j.procs.2015.12.067

[8] K. Wijaya, E.T. Baskoro, H. Assiyatun, D. Suprijanto, Subdivision of graphs in $\mathcal{R}\left(m K_{2}, P_{4}\right)$, Heliyon 6 (2020) e03843.

DOI: https://doi.org/10.1016/j.heliyon.2020.e03843

[9] K. Wijaya, E.T. Baskoro, H. Assiyatun, D. Suprijanto, On Ramsey $\left(m K_{2}, H\right)$-minimal graphs, Graphs Comb. 33 (1) (2017) 233-243.

DOI: https://doi.org/10.1007/s00373-016-1748

[10] E.T. Baskoro, L. Yulianti, On Ramsey minimal graphs for $2 K_{2}$ versus $P n$, Adv. Appl. Discrete Math. 8(2) (2011) 83-90. DOI http://www.pphmj.com/journals/articles/880.htm 